\title{
Risk Factors for the Development and Progression of Atlantoaxial Subluxation in Surgically Treated Rheumatoid Arthritis Patients, Considering the Time Interval between Rheumatoid Arthritis Diagnosis and Surgery
}

\author{
Min-Kyun Na, M.D., ${ }^{1}$ Hyoung-Joon Chun, M.D., Ph.D., ${ }^{1}$ Koang-Hum Bak, M.D., Ph.D., ${ }^{1}$ Hyeong-Joong Yi, M.D., Ph.D., ${ }^{1}$ Je II Ryu, M.D., ${ }^{2}$ \\ Myung-Hoon Han, M.D. ${ }^{2}$ \\ Department of Neurosurgery, ${ }^{1}$ Hanyang University Medical Center, Seoul , Korea \\ Department of Neurosurgery, ${ }^{2}$ Hanyang University Guri Hospital, Guri, Korea
}

\begin{abstract}
Objective : Rheumatoid arthritis (RA) is a systemic disease that can affect the cervical spine, especially the atlantoaxial region. The present study evaluated the risk factors for atlantoaxial subluxation (AAS) development and progression in patients who have undergone surgical treatment. Methods : We retrospectively analyzed the data of 62 patients with RA and surgically treated AAS between 2002 and 2015. Additionally, we identified 62 patients as controls using propensity score matching of sex and age among 12667 RA patients from a rheumatology registry between 2007 and 2015. We extracted patient data, including sex, age at diagnosis, age at surgery, disease duration, radiographic hand joint changes, and history of methotrexate use, and laboratory data, including presence of rheumatoid factor and the C-reactive protein (CRP) level.

Results : The mean patient age at diagnosis was 38.0 years. The mean time interval between RA diagnosis and AAS surgery was $13.6 \pm 7.0$ years. The risk factors for surgically treated AAS development were the serum CRP level $(p=0.005)$ and radiographic hand joint erosion $(p=0.009)$. The risk factors for AAS progression were a short time interval between RA diagnosis and radiographic hand joint erosion $(p<0.001)$ and young age at RA diagnosis $(p=0.04)$.

Conclusion : The CRP level at RA diagnosis and a short time interval between RA diagnosis and radiographic hand joint erosion might be risk factors for surgically treated AAS development in RA patients. Additionally, a short time interval between RA diagnosis and radiographic hand joint erosion and young age at RA diagnosis might be risk factors for AAS progression.
\end{abstract}

Key Words : Atlantoaxial subluxation · Rheumatoid arthritis · Posterior cervical fusion.

\section{INTRODUCTION}

Rheumatoid arthritis (RA) is a chronic systemic inflammatory disorder characterized by poly-articular synovial inflammation and progressive joint destruction ${ }^{3,25)}$, and it has been reported to affect approximately $0.5-1 \%$ of the adult population ${ }^{2}$. The cervical spine is commonly affected in patients with RA synovitis and enthesitis ${ }^{6,14,15)}$, and shows atlantoaxial subluxation (AAS), vertical subluxation (VS) of the axis, and subaxial subluxation (SAS). AAS is the most common type of cervical involvement, and the prevalence ranges from $19 \%$ to $70 \%$ of RA patients ${ }^{14,15,17)}$.
Chronic synovitis and erosive pannus formation at the joint may cause bony destruction and ligamentous laxity in the surrounding ligamentous complex, resulting in instability and subluxation $^{12,18)}$. The subluxation can be anterior, posterior, lateral, or rotatory. Anterior AAS is the most common subtype, with a prevalence of $10-55 \%^{7,8,17)}$. Many studies have reported the prevalence of cervical spine involvement and the predictive risk factors for rheumatoid cervical spine involvement, such as erosion at the peripheral joints, corticosteroid administration, early diagnosis of RA at $<45$ years of age, and presence of rheumatoid factor $(\mathrm{RF})^{1,10,23,24,26)}$. Because subluxation could result in irre-

- Received : May 30, 2016 • Revised : August 26, 2016 • Accepted : August 30, 2016

- Address for reprints : Hyoung-Joon Chun, M.D., Ph.D.

Department of Neurosurgery, Hanyang University Medical Center, 222-1 Wangsimni-ro, Seongdong-gu, Seoul 04763, Korea

Tel : +82-2-2290-8494, Fax : +82-2-2281-0954, E-mail : tdy815@hanyang.ac.kr

- This is an Open Access article distributed under the terms of the Creative Commons Attribution Non-Commercial License (http://creativecommons.org/licenses/by-nc/3.0) which permits unrestricted non-commercial use, distribution, and reproduction in any medium, provided the original work is properly cited. 
versible neural impairment, non-ambulation, respiratory dysfunction, and consequent death ${ }^{5)}$, its early diagnosis and treatment should be a priority in patients with RA.

The present study aimed to evaluate the risk factors for the development and progression of AAS in RA patients who have undergone surgical treatment, considering the time interval between RA diagnosis and AAS surgery. To our knowledge, this is the first such study in RA patients.

\section{MATERIALS AND METHODS}

\section{Patient data collection}

We retrospectively collected the data of 62 patients with RA and AAS from our hospital records between November 1, 2002 and February 28, 2015. All patients met the revised American College of Rheumatology criteria for $\mathrm{RA}^{3)}$ and had a known diagnosis of AAS [atlantodental interval $(\mathrm{ADI})>3 \mathrm{~mm}]^{20)}$. The patients experienced severe nuchal pain with upper extremity radiating pain. The detailed chief complains before surgery are presented in Table 1. All patients underwent surgery for posterior fusion. Of the 62 patients, 14 underwent unilateral transarticular screw fixation, 17 underwent bilateral transarticular screw fixation, 15 underwent unilateral $\mathrm{C} 1$ lateral mass and $\mathrm{C} 2$ pedicle screw fixation, 15 underwent bilateral C1 lateral mass and C2 pedicle screw fixation, and 1 underwent occipitocervical screw fixation. Furthermore, 48 patients underwent additional fusion with a wire using Sonntag's modified Gallie fusion technique (Table 1).

Our ongoing RA registry (established in 2007), which was designed for the purpose of research, includes 12667 RA patients ( $\geq 18$ years of age at RA diagnosis). Among these patients, we identified 1840 patients who underwent cervical radiography, including full flexion/extension view. We then excluded patients diagnosed with AAS and identified 966 patients as possible controls

Table 1. Characteristics of the surgically treated patients

\begin{tabular}{lc}
\multicolumn{1}{c}{ Cause and surgical techniqe } & $\begin{array}{c}\text { Total } \\
(\mathrm{n}=62)\end{array}$ \\
\hline Cause & \\
Spontaneous & $62(100.0 \%)$ \\
$\quad$ Suboccipital headache & $10(16.1 \%)$ \\
Nuchal pain & $27(43.6 \%)$ \\
Upper extremity paresthesia & $20(32.3 \%)$ \\
$\quad$ Upper extremity hypoesthesia & $3(4.8 \%)$ \\
$\quad$ Upper extremity motor weakness & $2(3.2 \%)$ \\
Trauma & $0(0.0 \%)$ \\
Surgical technique & \\
Unilateral transarticular screw fixation & $14(22.6 \%)$ \\
Bilateral transarticular screw fixation & $17(27.4 \%)$ \\
Unilateral C1 lateral mass and C2 pedicle screw fixation & $15(24.2 \%)$ \\
Bilateral C1 lateral mass and C2 pedicle screw fixation & $15(24.2 \%)$ \\
Occipitocervical screw fixation & $1(1.6 \%)$ \\
\hline
\end{tabular}

Data are presented as number (percentage) for the study. Among these patients, we finally identified 62 patients as controls, using propensity score matching of sex and age.

This study was approved by our institutional review board, and informed consent was not required owing to the retrospective nature of this study.

\section{Variables}

We reviewed the medical charts of all 62 patients surgically treated for AAS (surgery group) and all 62 matched controls (control group). We extracted patient data, including sex, age at RA diagnosis, age at AAS surgery, disease duration, and history of methotrexate (MTX) use, and laboratory data, including presence of RF (assessed at baseline) ${ }^{13)}$ and the C-reactive protein (CRP) level (measured at baseline).

\section{Image analysis}

For radiological evaluation of the cervical spine, we followed the classification system of Yurube ${ }^{25)}$. Cervical spine radiographs were obtained in anteroposterior and lateral views during full flexion and extension. Hyperflexion lateral view radiographs were used for evaluation of AAS. Anterior AAS was measured by recording the shortest distance between the posterior surface of the anterior arch of the atlas and the anterior surface of the odontoid process. VS was diagnosed according to the Ranawat value. SAS was diagnosed if a vertebra had moved more than 2 $\mathrm{mm}$ in relation to the next vertebra, when measured from the posterior line of the vertebral bodies. Radiological cervical findings for diagnosis were as follows : 1) AAS : ADI $>3 \mathrm{~mm}^{20,24)} ; 2$ ) VS : Ranawat value $<13 \mathrm{~mm}^{16)}$; and 3) SAS : irreducible translation $\geq 2 \mathrm{~mm}^{9}$. Additionally, radiological cervical findings for the diagnosis of severe conditions were as follows : 1) severe AAS : $\mathrm{ADI} \geq 10 \mathrm{~mm}^{15,24)}$; 2) severe VS : Ranawat value $\leq 10 \mathrm{~mm}^{16)}$; and 3) severe SAS : translation $\geq 4 \mathrm{~mm}$ or translation at multiple lev$\mathrm{els}^{9)}$. The presence of erosion was investigated using radiographs of the hands and feet in all patients.

\section{Statistical analysis}

Descriptive statistics were used to analyze the clinical information, demographic factors, and other data. Baseline characteristics of the patients are presented as mean \pm standard deviation and number/percentage. The chi-square and Student's t-test were used to assess differences between the surgery and matched groups.

We used the univariate and multivariate logistic regression analyses to evaluate the risk factors for surgically treated AAS development. The covariates were sex, age at RA diagnosis, RF positivity, MTX use, CRP level, and time interval between RA diagnosis and radiographic hand joint erosion.

We used the univariate linear regression analysis to evaluate the potential associations of variables with the time interval between RA diagnosis and AAS surgery. We then entered covariates into the multivariate analysis to identify the independent association of each variable with the time interval between RA 
diagnosis and AAS surgery.

Regression lines with $95 \%$ confidence intervals (CIs) and box plots were used to graphically present the associations of sex, time interval between RA diagnosis and hand joint erosion, age at surgery, and time interval between RA diagnosis and MTX initiation with the time interval between RA diagnosis and AAS surgery.

All statistical analyses were performed using R software (version 3.1.2; R Foundation for Statistical Computing, Vienna, Austria) and SPSS software for Windows (version 22.0; IBM Corp., Armonk, NY, USA). A $p$-value $<0.05$ was considered statistically significant.

\section{RESULTS}

The characteristics of the 124 study patients are presented in Table 2. The time interval between RA diagnosis and radiographic hand joint erosion was lower in the surgery group than in the matched group ( $2.9 \pm 2.9$ years vs. $4.0 \pm 3.3$ years, $p=0.042)$. Additionally, the CRP level at RA diagnosis was higher in the surgery group than in the matched group ( $4.0 \pm 3.7$ vs. $1.9 \pm 2.3, p<0.001)$. However, there were no significant differences in RF positivity and MTX use between the 2 groups. In the surgery group, the mean age of the patients at surgery was 52.1 years, and the mean time interval between RA diagnosis and AAS surgery was 13.6 years. Fig. 1 shows the number of surgically treated patients stratified into 6 categories based on a 5-year time interval between RA diagnosis and AAS surgery. Additionally, the mean time interval between RA diagnosis and radiographic hand joint erosion was 2.5 years.

To evaluate the total incidence of cervical spine subluxation, we assessed the patients according to "mild" and "severe" condi-

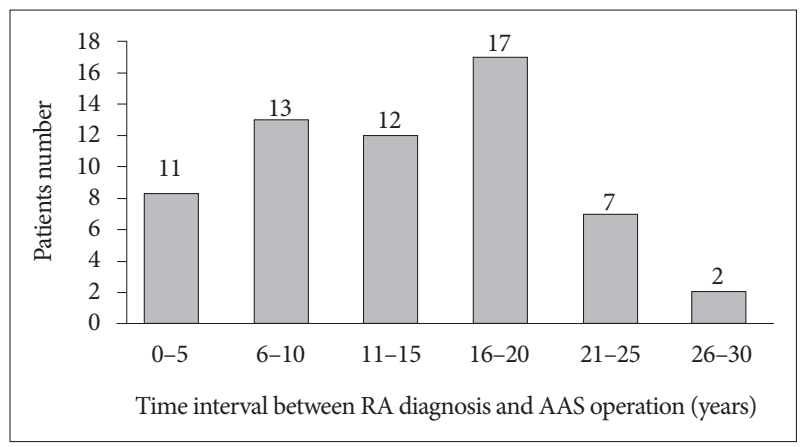

Fig. 1. The number of surgically treated patients stratified into 6 categories based on a 5-year time interval between RA diagnosis and AAS surgery. A total of $53(85.4 \%)$ patients underwent surgical treatment less than 20 years after RA diagnosis. RA : rheumatoid arthritis, AAS : atlantoaxial subluxation.

Table 2. Characteristics of the study patients

\begin{tabular}{|c|c|c|c|c|}
\hline Variables & $\begin{array}{c}\text { Total } \\
(\mathrm{n}=124)\end{array}$ & $\begin{array}{l}\text { Surgery group } \\
\quad(\mathrm{n}=62)\end{array}$ & $\begin{array}{l}\text { Matched group } \\
\quad(n=62)\end{array}$ & $p$-value \\
\hline Sex & & & & 1.000 \\
\hline Female & $113(91.1 \%)$ & $56(90.3 \%)$ & $57(91.9 \%)$ & \\
\hline Male & $11(8.9 \%)$ & $6(9.7 \%)$ & $5(8.1 \%)$ & \\
\hline Age at RA diagnosis, $y$ & $39.8 \pm 12.2$ & $38.0 \pm 11.9$ & $41.6 \pm 12.4$ & 0.098 \\
\hline Age at surgery, y & - & $52.1 \pm 12.6$ & - & - \\
\hline $\mathrm{RF}$ & & & & 0.685 \\
\hline Negative & $33(26.6 \%)$ & $18(29.0 \%)$ & $15(24.2 \%)$ & \\
\hline Positive & $91(73.4 \%)$ & $44(71.0 \%)$ & $47(75.8 \%)$ & \\
\hline MTX & & & & 0.680 \\
\hline No & $6(4.8 \%)$ & $2(3.2 \%)$ & $4(6.5 \%)$ & \\
\hline Yes & $118(95.2 \%)$ & $60(96.8 \%)$ & $58(93.5 \%)$ & \\
\hline Time interval between RA diagnosis and radiographic hand joint erosion, $y$ & $3.5 \pm 3.1$ & $2.9 \pm 2.9$ & $4.0 \pm 3.3$ & 0.042 \\
\hline $\mathrm{aADI}, \mathrm{mm}$ & $4.4 \pm 3.2$ & $7.1 \pm 2.4$ & $1.7 \pm 0.8$ & 0.000 \\
\hline \multicolumn{5}{|l|}{ aADI classification } \\
\hline $0.0-2.9$ & $62(50.0 \%)$ & - & $62(100.0 \%)$ & \\
\hline $3.0-5.0$ & $12(9.7 \%)$ & $12(19.4 \%)$ & - & \\
\hline $5.1-7.5$ & $27(21.8 \%)$ & $27(43.5 \%)$ & - & \\
\hline $7.6-10.0$ & $15(12.1 \%)$ & $15(24.2 \%)$ & - & \\
\hline$>10.0$ & $8(6.4 \%)$ & $8(12.9 \%)$ & - & \\
\hline CRP level, mg/dL & $2.9 \pm 3.2$ & $4.0 \pm 3.7$ & $1.9 \pm 2.3$ & 0.000 \\
\hline Time interval between RA diagnosis and AAS surgery, $y$ & - & $13.6 \pm 7.0$ & - & - \\
\hline Time interval between RA diagnosis and AAS diagnosis, $y$ & - & $11.0 \pm 6.9$ & - & - \\
\hline Time interval between AAS diagnosis and AAS surgery, $y$ & - & $2.5 \pm 2.7$ & - & - \\
\hline
\end{tabular}

Data are presented as mean \pm standard deviation or number (percentage). RF : rheumatoid factor, RA : rheumatoid arthritis, MTX : methotrexate, aADI : anterior atlantodental interval, CRP : C-reactive protein, AAS : atlantoaxial subluxation 
tions. We found that $100 \%$ of the patients had AAS, $17.8 \%$ had VS, $29.1 \%$ had SAS, and $40.3 \%$ had basilar invagination, with some overlap. There was no evidence of severity associations among instabilities, and we found that all severe SAS patients had mild AAS (Table 3).
In multivariate logistic regression analysis, age at RA diagnosis [odds ratio (OR), 0.97; 95\% CI, 0.94-1.00; $p=0.049$ ], CRP level (OR, 3.21; 95\% CI, 1.41-7.30; $p=0.005$ ), and time interval between RA diagnosis and radiographic hand joint erosion (OR, 0.83 ; 95\% CI, 0.72-0.96; $p=0.009$ ) were identified as risk factors
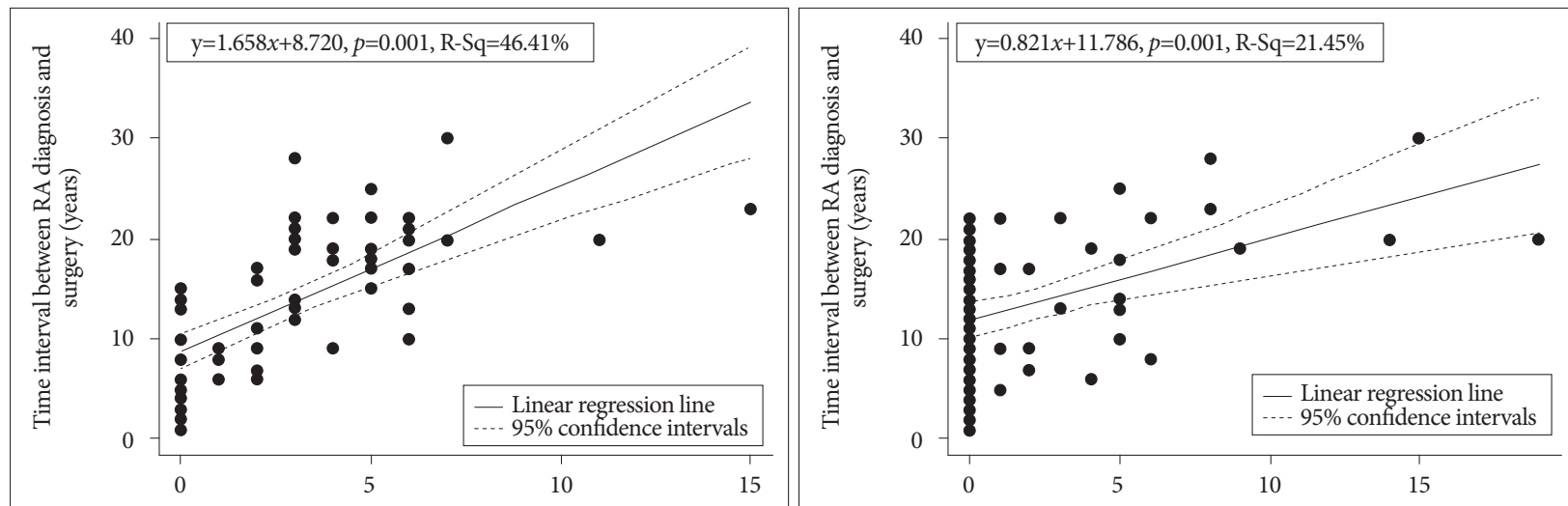

(A) Time interval between RA diagnosis and hand joint erosion

(B) Time interval between RA diagnosis and MTX initiation

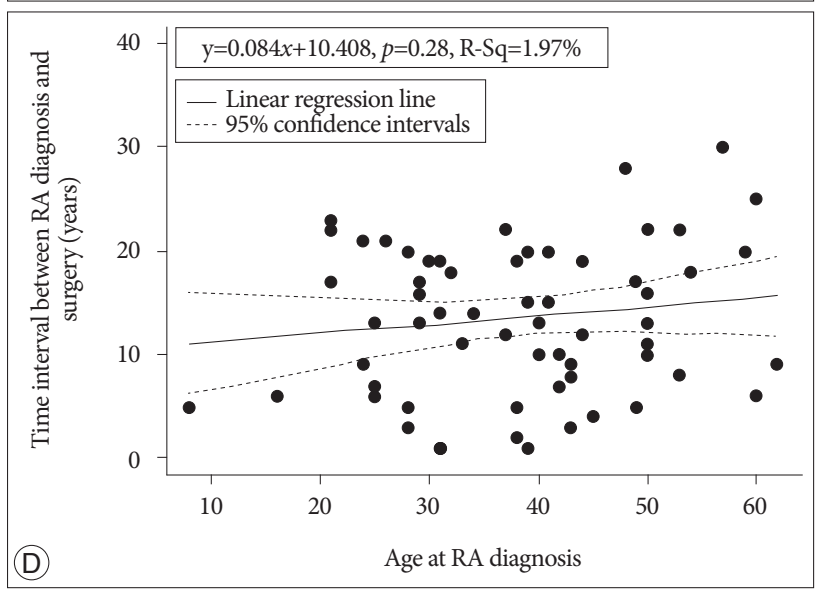

Fig. 2. A : In scatterplot graph, the linear regression line shows a statistically significant overall gradual upward slope, which means the time to surgery reduces by 1.658 years when the time to radiographic hand joint erosion reduces by 1 year. $\mathrm{B}:$ In scatterplot graph, the linear regression line shows a statistically significant overall gradual upward slope, indicating that patients who started MTX early required early surgery. C : The boxplot graph shows the mean time interval between RA diagnosis and AAS surgery was 4.7 years greater in female patients than in male patients. D : In scatterplot graph, the linear regression line shows an upward trend, indicating that patients who are diagnosed with RA at a young age might require early AAS surgery. RA : rheumatoid arthritis, AAS : atlantoaxial subluxation.

Table 3. Descriptive statistics according to the atlantoaxial subluxation subgroups

\begin{tabular}{|c|c|c|c|c|}
\hline Atlantoaxial subluxation & Total $(\mathrm{n}=62)$ & Mild $(\mathrm{aADI} \leq 10 \mathrm{~mm})(\mathrm{n}=54)$ & Severe $(\mathrm{aADI}>10 \mathrm{~mm})(\mathrm{n}=8)$ & $p$-value \\
\hline Vertical subluxation & $11(17.8 \%)$ & & & 0.838 \\
\hline No & $51(82.2 \%)$ & $44(81.5 \%)$ & $7(87.5 \%)$ & \\
\hline Mild $(10<$ ranawat value $\leq 13)$ & $9(14.5 \%)$ & $8(14.8 \%)$ & $1(12.5 \%)$ & \\
\hline Severe (ranawat value $\leq 10$ ) & $2(3.3 \%)$ & $2(3.7 \%)$ & $0(0.0 \%)$ & \\
\hline Subaxial subluxation & $18(29.1 \%)$ & & & 0.153 \\
\hline No & $44(70.9 \%)$ & $36(66.7 \%)$ & $8(100.0 \%)$ & \\
\hline Mild $(2 \leq$ irreducible translation $<4)$ & $14(22.6 \%)$ & $14(25.9 \%)$ & $0(0.0 \%)$ & \\
\hline Severe (irreducible translation $\geq 4$ ) & $4(6.5 \%)$ & $4(7.4 \%)$ & $0(0.0 \%)$ & \\
\hline Basilar invagination & $25(40.3 \%)$ & & & 0.575 \\
\hline No & $37(59.7 \%)$ & $31(57.4 \%)$ & $6(75.0 \%)$ & \\
\hline Yes & $25(40.3 \%)$ & $23(42.6 \%)$ & $2(25.0 \%)$ & \\
\hline
\end{tabular}

Data are presented as number (percentage). aADI : anterior atlantodental interval 
Table 4. Risk factors to predict surgically treated AAS development in patients with rheumatoid arthritis

\begin{tabular}{|c|c|c|c|c|}
\hline \multirow{2}{*}{ Variable } & \multicolumn{2}{|c|}{ Univariate analysis } & \multicolumn{2}{|c|}{ Multivariate analysis } \\
\hline & OR $(95 \% \mathrm{CI})$ & $p$ & OR $(95 \% \mathrm{CI})$ & $p$ \\
\hline Sex $($ reference $=$ male $)$ & $0.82(0.24-2.84)$ & 0.752 & $0.91(0.24-3.45)$ & 0.888 \\
\hline Age at RA diagnosis (per 1-year increase) $)^{*}$ & $0.98(0.95-1.01)$ & 0.099 & $0.97(0.94-1.00)$ & 0.049 \\
\hline RF positivity & $0.78(0.35-1.74)$ & 0.543 & $0.67(0.27-1.65)$ & 0.382 \\
\hline MTX use & $2.07(0.37-11.73)$ & 0.412 & $2.86(0.43-19.18)$ & 0.280 \\
\hline CRP level $(>0.8 \mathrm{mg} / \mathrm{dL})$ & $2.70(1.27-5.74)$ & 0.010 & $3.21(1.41-7.30)$ & 0.005 \\
\hline Time interval (per 1-year increase)* & $0.88(0.78-0.99)$ & 0.047 & $0.83(0.72-0.96)$ & 0.009 \\
\hline
\end{tabular}

${ }^{*}$ Time interval between RA diagnosis and radiographic hand joint erosion. RA : rheumatoid arthritis, AAS : atlantoaxial subluxation, OR : odds ratio, $\mathrm{Cl}$ : confidence interval, RF : rheumatoid factor, MTX : methotrexate, CRP : C-reactive protein

Table 5. Univariate and multivariate linear regression analyses of the associations of various patient factors with the time interval between RA diagnosis and AAS surgery

\begin{tabular}{|c|c|c|c|c|c|c|c|c|}
\hline \multirow{2}{*}{ Variable } & \multicolumn{3}{|c|}{ Univariate linear regression } & \multicolumn{5}{|c|}{ Multivariate linear regression } \\
\hline & $\beta$ & SE & $p$ & Intercept & $\beta$ & SE & $p$ & $\mathrm{R}^{2}$ \\
\hline Sex $($ reference $=$ male $)$ & 4.738 & 2.988 & 0.118 & \multirow{7}{*}{0.498} & 4.634 & 2.287 & 0.048 & \multirow{7}{*}{0.568} \\
\hline Age at RA diagnosis & 0.084 & 0.076 & 0.277 & & 0.125 & 0.060 & 0.044 & \\
\hline $\mathrm{RF}$ & 1.725 & 1.974 & 0.386 & & 2.384 & 1.487 & 0.115 & \\
\hline Time interval between RA diagnosis and MTX initiation & 0.821 & 0.203 & $<0.001$ & & 0.276 & 0.199 & 0.172 & \\
\hline $\begin{array}{l}\text { Time interval between RA diagnosis and radiographic } \\
\text { hand joint erosion }\end{array}$ & 1.658 & 0.230 & $<0.001$ & & 1.470 & 0.270 & $<0.001$ & \\
\hline $\mathrm{aADI}$ & -0.289 & 0.383 & 0.454 & & -0.282 & 0.278 & 0.314 & \\
\hline CRP level & 0.328 & 0.242 & 0.180 & & -0.121 & 0.194 & 0.534 & \\
\hline
\end{tabular}

RA : rheumatoid arthritis, AAS : atlantoaxial subluxation, SE : standard error, R2 : coefficient of determination, RF : rheumatoid factor, MTX : methotrexate, aADI : anterior atlantodental interval, CRP : C-reactive protein

for surgically treated AAS development in RA patients (Table 4).

Fig. $2 \mathrm{~A}$ presents a scatterplot for the time interval between RA diagnosis and hand joint erosion and the time interval between RA diagnosis and AAS surgery for all patients, with a linear regression line with 95\% CIs. The linear regression line shows a statistically significant overall gradual upward slope $(\beta=1.658$, $\mathrm{SE}=0.230, p<0.001$ ), and the time to surgery reduces by 1.658 years when the time to radiographic hand joint erosion reduces by 1 year, indicating that patients with early hand joint erosion required early surgery. Fig. $2 \mathrm{~B}$ presents a scatterplot for the time interval between RA diagnosis and MTX initiation and the time interval between RA diagnosis and AAS surgery for all patients, with a linear regression line with 95\% CIs. The linear regression line shows a statistically significant overall gradual upward slope $(\beta=0.821, \mathrm{SE}=0.203, p<0.001)$, indicating that patients who started MTX early required early surgery.

In multivariate linear regression analysis, after complete adjustment, the time interval between RA diagnosis and radiographic hand joint erosion $(\beta=1.470, \mathrm{SE}=0.270, p<0.001)$, age at RA diagnosis $(\beta=0.125, \mathrm{SE}=0.060, p=0.044)$, and sex $(\beta=4.634$, $\mathrm{SE}=2.287, p=0.048)$ were identified as risk factors for AAS progression (Table 5). However, the time interval between RA diagnosis and MTX initiation ( $p=0.172$ ), RF seropositivity ( $p=0.115$ ), the serum level of CRP $(p=0.534)$, and the anterior $\operatorname{ADI}(p=0.314)$ were not significantly associated with disease progression (Table 5). Fig. 2C presents a boxplot for sex and the time interval between RA diagnosis and AAS surgery. We found that the mean time interval between RA diagnosis and AAS surgery was 4.7 years greater in female patients than in male patients. Fig. $2 \mathrm{D}$ presents a scatterplot for age at RA diagnosis and the time interval between RA diagnosis and AAS surgery. The linear regression line shows an upward trend $(\beta=0.084, \mathrm{SE}=0.076, p=0.277)$, indicating that patients who are diagnosed with RA at a young age might require early AAS surgery.

\section{DISCUSSION}

RA is a chronic systemic inflammatory disease, and cervical spine involvement may cause severe complications, such as headache, neck pain, upper extremity paresthesia, and death ${ }^{7,19)}$. The prevalence of cervical spine involvement has been shown to range from $16 \%$ to $70.4 \%$, and AAS has been reported to be the most common abnormal finding ${ }^{1,4,24)}$. The risk factors for cervical involvement have been shown to be peripheral hand or foot joint erosion $^{1,4,24)}$, disease-modifying antirheumatic drug (DMARD) failure $^{4)}$, corticosteroid use ${ }^{24)}$, and age at RA diagnosis ${ }^{1)}$. Additionally, our study found that the CRP level and time interval between RA diagnosis and radiographic hand joint erosion were risk factors for surgically treated AAS development.

The natural course of conservatively treated rheumatoid patients with myelopathy is poor ${ }^{15,21)}$, and surgical decompression with fusion should be considered in such patients. Our study identified a short time interval between RA diagnosis and radiographic hand joint erosion and young age at RA diagnosis as 
risk factors for early surgical treatment. We believe that this is the first study to evaluate the risk factors for AAS progression in surgically treated RA patients, considering the time interval between RA diagnosis and AAS surgery.

A short time interval between RA diagnosis and radiographic hand joint erosion was associated with surgically treated AAS development $(p=0.009)$ in RA patients and progression $(p<0.001)$ in patients with surgically treated AAS. Previous studies have shown that radiographic hand and foot joint erosion in radiographs was the most common risk factor for cervical involvement in RA patients ${ }^{1,4,10,24)}$. Proliferative and erosive synovitis progresses to destruction of the articular cartilage, especially in metatarsophalangeal joints, which are the most commonly involved joints ${ }^{10)}$, and the cervical spine is the second most commonly involved region. The same pathophysiology affects the hand joints and upper cervical region, and patients with hand joint erosion tend to have cervical involvement and early progression of subluxation.

We noted a significant association between young age at RA diagnosis and early AAS surgery $(p=0.044)$. A previous study showed that RA diagnosis at $\leq 45$ years of age was a risk factor for cervical involvement ${ }^{1)}$. Although patients with RA diagnosis at a young age received conservative treatment, including combination therapy of DMARDs and corticosteroids, this treatment could not protect against the progression of the disease after the start of erosive changes.

The time interval between RA diagnosis and AAS surgery was lower in male patients than in female patients $(p=0.048)$, although the number of female patients was significantly greater than the number of male patients. However, the small number of male patients in the study might lower the statistical power, and there is a possibility that these findings are incidental owing to the small sample size.

In univariate analysis, the time interval between RA diagnosis and MTX initiation was associated with the time interval between RA diagnosis and AAS surgery $(p<0.001)$; however, in multivariate analysis, it was not a significant risk factor for early surgery $(p=0.172)$. The time interval between RA diagnosis and hand joint erosion and the time interval between RA diagnosis and MTX initiation were associated, indicating that patients with early hand joint erosion received MTX early $(\mathrm{R}=0.44, p=0.00$, Pearson correlation analysis). Therefore, if a patient has a severe condition at RA diagnosis, combination medication, including MTX, is initiated. A previous study reported that adequate treatment with DMARDs and biological agents (BAs) prevented the development of cervical spine disease, but probably did not prevent the progression of pre-existing injury ${ }^{10)}$. Additionally, another study reported that among patients who were administered MTX, only $8.3 \%$ without baseline instability developed instability, while $80 \%$ with baseline instability showed progression of instability ${ }^{1124)}$. Therefore, MTX can protect against the new onset of erosive synovitis; however, MTX cannot delay the progression of instability once destruction has started.
We found that the RF seropositivity was not a potential risk factor for surgically treated AAS development $(p=0.382)$ in RA patients and early surgical treatment $(p=0.115)$ in patients with surgically treated AAS. However, a previous study has noted RF positivity as a risk factor for disease progression ${ }^{7}$. The serum CRP level was a significant risk factor for surgically treated AAS development $(p=0.005)$ in RA patients, while it was not associated with disease progression in surgically treated AAS patients. Previous studies have identified a high serum CRP level as a risk factor for disease progression, and the CRP level was previously found to be very sensitive to changes in disease activity ${ }^{7,22)}$.

The present study has several limitations. As this was a retrospective study, the findings may not be as accurate as the findings of a prospective study. In addition, owing to the small number of patients in our study and the data heterogeneity between the surgery group and control group because of data extraction from different databases at different periods, there might be low statistical power. All age ranges were included in the surgery group and patients aged $\geq 18$ years were included in the control group. Moreover, this study focused on MTX, and other treatments, such as other DMARDs, BA, and corticosteroids, were not assessed. In future studies, larger number of patients should be included to confirm the risk factors for the prevalence of cervical involvement and progression of subluxation. Additionally, other medications should be assessed to determine their effects on disease progression.

\section{CONCLUSION}

The CRP level at RA diagnosis and a short time interval between RA diagnosis and radiographic hand joint erosion might be risk factors for surgically treated AAS development in RA patients. Additionally, a short time interval between RA diagnosis and radiographic hand joint erosion and young age at RA diagnosis might be risk factors for rapid progression of AAS. Immediately after RA diagnosis, patients should be treated with DMARDs and corticosteroids before radiographic changes are noted in the hand joints and cervical spine, especially at a young age. Examinations, such as radiographic evaluation of the peripheral joints and cervical spine and evaluation of inflammatory markers, should be performed annually in patients with RA to assess the development of cervical spine instability, which will help in treatment.

\section{- Acknowledgements}

This work was supported by the research fund of Hanyang University (HY-2012-G). The English grammar was revised by editage, Inc.

\section{References}

1. Ahn JK, Hwang JW, Oh JM, Lee J, Lee YS, Jeon CH : Risk factors for development and progression of atlantoaxial subluxation in Korean patients with rheumatoid arthritis. Rheumatol Int 31 : 1363-1368, 2011

2. Alamanos Y, Drosos AA : Epidemiology of adult rheumatoid arthritis. 
Autoimmun Rev $4:$ 130-136, 2005

3. Arnett FC, Edworthy SM, Bloch DA, McShane DJ, Fries JF, Cooper NS, et al. : The American Rheumatism Association 1987 revised criteria for the classification of rheumatoid arthritis. Arthritis Rheum 31 : 315-324, 1988

4. Blom M, Creemers MC, Kievit W, Lemmens JA, van Riel PL : Longterm follow-up of the cervical spine with conventional radiographs in patients with rheumatoid arthritis. Scand J Rheumatol $42: 281-288,2013$

5. Casey AT, Crockard HA, Bland JM, Stevens J, Moskovich R, Ransford $\mathrm{AO}$ : Surgery on the rheumatoid cervical spine for the non-ambulant myelopathic patient-too much, too late? Lancet 347 : 1004-1007, 1996

6. Delamarter RB, Bohlman HH : Postmortem osseous and neuropathologic analysis of the rheumatoid cervical spine. Spine (Phila Pa 1976) 19: 2267-2274, 1994

7. Fujiwara K, Fujimoto M, Owaki H, Kono J, Nakase T, Yonenobu K, et al. : Cervical lesions related to the systemic progression in rheumatoid arthritis. Spine (Phila Pa 1976) 23 : 2052-2056, 1998

8. Hirano K, Imagama S, Oishi Y, Kanayama Y, Ito Z, Wakao N, et al. : Progression of cervical instabilities in patients with rheumatoid arthritis 5.7 years after their first lower limb arthroplasty. Mod Rheumatol 22 : 743749,2012

9. Ito H, Neo M, Sakamoto T, Fujibayashi S, Yoshitomi H, Nakamura T : Subaxial subluxation after atlantoaxial transarticular screw fixation in rheumatoid patients. Eur Spine J 18 : 869-876, 2009

10. Joaquim AF, Appenzeller S2 : Cervical spine involvement in rheumatoid arthritis--a systematic review. Autoimmun Rev 13 : 1195-1202, 2014

11. Kaito T, Hosono N, Ohshima S, Ohwaki H, Takenaka S, Fujiwara H, et al. : Effect of biological agents on cervical spine lesions in rheumatoid arthritis. Spine (Phila Pa 1976) 37 : 1742-1746, 2012

12. Konttinen YT, Bergroth V, Santavirta S, Sandelin J : Inflammatory involvement of cervical spine ligaments in patients with rheumatoid arthritis and atlantoaxial subluxation. J Rheumatol $14: 531-534,1987$

13. Mathews JA : Atlanto-axial subluxation in rheumatoid arthritis. A 5-year follow-up study. Ann Rheum Dis 33 : 526-531, 1974

14. Naranjo A, Carmona L, Gavrila D, Balsa A, Belmonte MA, Tena X, et al. : Prevalence and associated factors of anterior atlantoaxial luxation in a nation-wide sample of rheumatoid arthritis patients. Clin Exp Rheu- matol 22 : 427-432, 2004

15. Oda T, Fujiwara K, Yonenobu K, Azuma B, Ochi T : Natural course of cervical spine lesions in rheumatoid arthritis. Spine (Phila Pa 1976) 20 : 1128-1135, 1995

16. Ranawat CS, O'Leary P, Pellicci P, Tsairis P, Marchisello P, Dorr L : Cervical spine fusion in rheumatoid arthritis. J Bone Joint Surg Am 61 : 10031010, 1979

17. Raskin RJ, Schnapf DJ, Wolf CR, Killian PJ, Lawless OJ : Computerized tomography in evaluation of atlantoaxial subluxation in rheumatoid arthritis. J Rheumatol $10: 33-41,1983$

18. Reiter MF, Boden SD : Inflammatory disorders of the cervical spine. Spine (Phila Pa 1976) 23 : 2755-2766, 1998

19. Renna R, Plantone F, Plantone D : Atlantoaxial subluxation in rheumatoid arthritis. J Rheumatol $40: 1925,2013$

20. Sharp J, Purser DW : Spontaneous atlanto-axial dislocation in ankylosing spondylitis and rheumatoid arthritis. Ann Rheum Dis 20 : 47-77, 1961

21. van Asselt KM, Lems WF, Bongartz EB, Hamburger HL, DrossaersBakker KW, Dijkmans BA, et al. : Outcome of cervical spine surgery in patients with rheumatoid arthritis. Ann Rheum Dis 60 : 448-452, 2001

22. van der Heijde DM, van't Hof MA, van Riel PL, van Leeuwen MA, van Rijswijk MH, van de Putte LB : Validity of single variables and composite indices for measuring disease activity in rheumatoid arthritis. Ann Rheum Dis 51 : 177-181, 1992

23. Winfield J, Cooke D, Brook AS, Corbett M : A prospective study of the radiological changes in the cervical spine in early rheumatoid disease. Ann Rheum Dis 40 : 109-114, 1981

24. Yurube T, Sumi M, Nishida K, Miyamoto H, Kohyama K, Matsubara T, et al. : Accelerated development of cervical spine instabilities in rheumatoid arthritis : a prospective minimum 5-year cohort study. PLoS One 9 : e88970, 2014

25. Yurube T, Sumi M, Nishida K, Miyamoto H, Kohyama K, Matsubara T, et al. : Incidence and aggravation of cervical spine instabilities in rheumatoid arthritis : a prospective minimum 5-year follow-up study of patients initially without cervical involvement. Spine (Phila Pa 1976) 37 : 2136-2144, 2012

26. Zhang T, Pope J : Cervical spine involvement in rheumatoid arthritis over time : results from a meta-analysis. Arthritis Res Ther 17 : 148, 2015 